\title{
INTEGRAL REPRESENTATIONS OF UNIVALENT FUNCTIONS AND SINGULAR MEASURES
}

\author{
ROBERT J. BASS
}

(Communicated by Clifford J. Earle, Jr.)

\begin{abstract}
In [5], T. MacGregor showed that not every univalent function has a proposed integral representation with respect to Borel measures on the unit circle. In this paper, we study the decomposition of measures which do give rise to univalent functions. The main results show that no measure with a continuous singular component can ever be associated with a univalent function.
\end{abstract}

\section{INTRODUCTION}

Let $\Delta=(z \in C:|z|<1)$ and $T=\partial \Delta$.

$M(T)$ will denote the set of finite Borel measures on $T ; m$ will denote normalized Lebesgue measure.

Finally, $M(T) /\left(H_{0}^{1}\right)^{-}$will denote the coset space generated by the closed subspace

$$
\left(H_{0}^{1}\right)^{-}=\left\{\mu \in M(T): \int_{T} x^{-n} d \mu(x)=0, \forall n \geq 1\right\},
$$

$\left(H_{0}^{1}\right)^{-}$is the annihilator of $A(T)=C^{0}(T) \cap H^{2}(T)$ in $M(T)=C^{0}(T)^{*}$. Therefore, $M(T) /\left(H_{0}^{1}\right)^{-}$can naturally be associated with the dual of $A(T)$. By the F. and M. Riesz theorem,

$$
\left(H_{0}^{1}\right)^{-}=\left\{\overline{f(x)} m(x): f \in H^{1}(T), \int_{T} f(x) d m(x)=0\right\} .
$$

In [5], MacGregor restated as follows a question regarding the linear span of the close-to-convex functions in the univalent functions on the unit disk:

If $f$ is analytic and univalent in $\Delta$, does $f$ have to be represented as in (1)?

$$
f(z)=l[\mu] \equiv \int_{T} \frac{1}{(1-\bar{x} z)^{2}} d \mu(x), \quad|z|<1,
$$

for some $\mu \in M(T)$. He resolved the question in the negative by a difficult linear-functional argument based on an idea of Baernstein.

Received by the editors August 7, 1989.

1980 Mathematics Subject Classification (1985 Revision). Primary 30E20; Secondary 30C45.

Key words and phrases. Univalent functions, integral representations, close-to-convex functions. 
In this paper, we shall consider the following related question: If $l[\mu]$ is univalent, what can be deduced about the measure $\mu$ ?

We first observe that any representation such as (1) is necessarily not unique. The $n$th Taylor coefficient of $l[\mu]$,

$$
\frac{l[\mu]^{n}(0)}{n !}=(n+1) \int_{T} x^{-n} d \mu(x) .
$$

Therefore, $l[\mu]=l[\nu]$ if and only if $(\mu-\nu) \in\left(H_{0}^{1}\right)^{-}$(i.e., $[\mu]=[\nu] \epsilon$ $\left.M(T) /\left(H_{0}^{1}\right)^{-}\right)$. In that case, their components which are singular with respect to Lebesgue measure, $\mu_{s}$ and $\nu_{s}$, are equal. Therefore, the appropriate question is as follows:

If $l[\mu]$ is univalent, what can be deduced about $\mu_{s}$ ?

Example. Consider the Koebe function

$$
\begin{aligned}
k(z) & =\frac{z}{(1-z)^{2}}=\frac{1}{(1-z)^{2}}-\frac{1}{(1-z)} \\
& =\frac{1}{(1-z)^{2}}-\int_{T} \frac{1}{(1-\bar{x} z)^{2}} \log \left(\frac{1}{1-x}\right) d m(x) \\
& =l\left(\delta_{1}-\log \left(\frac{1}{1-x}\right) d m(x)\right)(z),
\end{aligned}
$$

where $\delta_{\zeta}$ is the unit point mass at $\zeta \in T$. Thus, there is at least one singular measure $\mu$ which gives rise to a univalent function $l[\mu]$.

The main result of this paper is that the Koebe example represents the exceptional case. We shall prove the following:

Theorem A. Let $l[\mu]$ be univalent. Then

(i) there is at most one point of mass for the measure $\mu$ (i.e., $\mu(\{\zeta\})=0$ for all but at most one point $\zeta \in T)$.

(ii) the continuous component of $\mu$ ( $\mu$ minus its point masses) is absolutely continuous.

Along the way, we will prove a similar result for the Cauchy-Stieltjes integral of a measure:

$$
C[\mu](z)=\int_{T} \frac{1}{(1-\bar{x} z)} d \mu(z), \quad|z|<1 .
$$

Theorem B. Let $C[\mu]$ be univalent. Then

(i) there are at most two points of mass for the measure $\mu$.

(ii) the continuous component of $\mu$ is absolutely continuous.

\section{LOCAL $H^{p}$ PROPERTIES OF UNIVALENT FUNCTIONS}

We begin by proving some local Hardy space smoothness properties for univalent functions. These are likely to be well known, or at least not unexpected. We include them here for completeness. 
Recall that for a general domain $\Omega$, a function $f$, analytic on $\Omega$, is in the space $H^{p}(\Omega)$ if there exists $u$, harmonic on $\Omega$, such that

$$
|f(z)|^{p} \leq u(z), \quad \forall z \in \Omega .
$$

Definition. Let $\Omega$ be a smooth, Jordan domain, $\zeta \in \partial \Omega$, and $f$ be analytic on $\Omega$. We will say that $f$ is locally $H^{p}$ at $\zeta$, denoted $H_{\text {loc }}^{p}(\Omega ; \zeta)$, if there exist $\varepsilon>0$ and a harmonic function $u_{\zeta, \varepsilon}$ defined in

$$
\Omega(\zeta, \varepsilon) \equiv \Omega \cap\{z:|z-\zeta|<\varepsilon\},
$$

such that

$$
|f(z)|^{p}<u_{\zeta, \varepsilon}(z), \quad \forall z \in \Omega(\zeta, \varepsilon) .
$$

For an arc $l$ in $\partial \Omega$, we will say that $f \in H_{\mathrm{loc}}^{p}(\Omega ; l)$ if $f \in H_{\mathrm{loc}}^{p}(\Omega ; \zeta)$ for every $\zeta \in l$.

The key results on local $H^{p}$ are due to Gauthier and Hengartner [3]. In the notation set up above, we state one of their results as follows:

$$
H_{\mathrm{loc}}^{p}(\Omega ; \partial \Omega)=H^{p}(\Omega) .
$$

We now localize some standard results about univalent functions.

Proposition A. Let $f$ be univalent in $\Delta, G^{\prime}=f, G(0)=0$. Then

(i) $f \in H_{\text {loc }}^{1 / 2}(\Delta ; \zeta)$ for all but at most one point, $\zeta \in T$.

(ii) $f \in H_{\mathrm{loc}}^{1}(\Delta ; \zeta)$ for all but at most two points, $\zeta \in T$.

(iii) $G \in H_{\mathrm{loc}}^{1}(\Delta ; \zeta)$ for all but at most one point, $\zeta \in T$.

(iv) For every $p<\infty, G \in H_{\mathrm{loc}}^{p}(\Delta ; \zeta)$ for all but at most two points, $\zeta \in T$.

Before proving the proposition, we recall Hayman's concept of the order of a univalent function at a boundary point [4] and Prawitz's inequality.

Let $f$ be univalent. Assume $f(0)=0$, so that $0 \notin f(T)$.

Definition. The order of $f$ at $\zeta \in T$ is given by

$$
\begin{aligned}
o(f ; \zeta)=\sup \{\delta: \exists \gamma:[0, \infty) \rightarrow \Delta, & \gamma(t) \stackrel{t \rightarrow \infty}{\longrightarrow} \zeta, \\
& \left.\ni \frac{\lim }{t}(1-|\gamma(t)|)^{\delta}|f(\gamma(t))|>0\right\} .
\end{aligned}
$$

It is trivial to see that this definition is equivalent to

$$
o(f ; \zeta)=\inf \left\{\beta: \lim _{z \rightarrow \zeta, z \in \Delta}(1-|z|)^{\beta}|f(z)|=0\right\} .
$$

Theorem (Hayman) [4, p. 27]. $o(f ; \zeta)=0$ for all but at most a countable number of points $\zeta \in T$. Moreover,

$$
\sum_{\zeta \in T} o(f ; \zeta) \leq 2
$$

The following is essentially a restatement of the Prawitz inequality (see, for example, [2]). 
Lemma. Let $M=\max \{o(f ; \zeta): \zeta \in T\}$. Then $f \in H^{p}$, for all $p<1 / M$.

Proof. Let $\varepsilon>0$ be given. By a compactness argument, under the stated assumptions

$$
M_{\infty}(r, f) \equiv \sup _{|z|=r}|f(z)|=o\left(\frac{1}{(1-r)^{M+\varepsilon}}\right) .
$$

Therefore, by the Prawtiz inequality,

$$
\begin{aligned}
\int_{T}|f(r x)|^{p} d m(x) & \leq p \int_{0}^{r} \frac{\left(M_{\infty}(t, f)\right)^{p}}{t} d t \\
& \leq C \int_{0}^{1} \frac{1}{(1-t)^{p(M+\varepsilon)}} d t \\
& <\infty, \quad \text { if } p(M+\varepsilon)<1 .
\end{aligned}
$$

This is completely familiar; however, we now set up a localized form.

Let $l$ be a closed arc in $T$ and $\varepsilon>0$ be fixed. Let $k l$ denote the arc with the same center as $l$ and $m(k l)=k m(l)$. Let

$$
M_{l} \equiv \max _{\zeta \in(1+\varepsilon) l} o(f ; \zeta) \text {. }
$$

(Notice that if $\varepsilon$ is sufficiently small and $M_{l} \neq 0$, then Hayman's theorem guarantees that $M_{l}$ is independent of $\varepsilon$ ).

\section{Proposition B.}

$$
f \in H_{\mathrm{loc}}^{p}(\Delta ; l), \quad \forall p<1 / M_{l} .
$$

Proof. Let $\Omega_{l}$ be a $C^{\infty}$, convex domain contained in $\Delta$, such that $\partial \Omega_{l} \cap T=$ $(1+\varepsilon) l$, and $0 \in \Omega_{l}$. Let $\phi$ be a Riemann map onto $\Omega_{l}$ such that $\phi(0)=0$.

By the smoothness of $\phi, \phi^{-1}$, and $\phi^{\prime}$, it is easy to see that

$$
o\left(f(\phi) ; \phi^{-1}(\zeta)\right)=o(f ; \zeta), \quad \forall \zeta \in(1+\varepsilon) l .
$$

Moreover, if $\lambda \in \phi^{-1}\left(\partial \Omega_{\nearrow} \backslash(1+\varepsilon) l\right), f(\phi)$ is continuous at $\lambda$, so that

$$
o(f ; \lambda)=0 \text {. }
$$

Therefore,

$$
\max _{\lambda \in T} o(f ; \lambda)=M_{l}
$$

and

$$
f(\phi) \in H^{p}(\Delta), \quad \forall p<\frac{1}{M_{l}} .
$$

There then exists a harmonic majorant $u$ for $|f(\phi)|^{p}$ :

$$
|f(\phi(w))|^{p} \leq u(w) .
$$

So $u\left(\phi^{-1}\right)$ serves as a local harmonic majorant of $|f|^{p}$ at each point $\zeta \in l$.

The proofs of Proposition A (i) and (ii) now follow by observing that Hayman's theorem allows at most one point $\zeta$ where $o(f ; \zeta)=2$ and at most 
two points $\zeta_{1}, \zeta_{2}$ where $o\left(f ; \zeta_{j}\right) \geq 1$. Parts (iii) and (iv) are likewise then localized versions of the following (see, e.g., [1]).

(1) If $g \in H^{1 / 2}$, then $\int^{z} g \in H^{1}$.

(2) If $g \in H^{1}$, then $\int^{z} g \in H^{p}$ for every $p<\infty$.

2. LOCAL WEAK-* CONVERGENCE IN $M(T) /\left(H_{0}^{1}\right)^{-}$

Lemma. Let $F=\int^{z} f$. Then $f=l[\mu]$ if and only if $F=C[\mu]-C[\mu](0)$.

Proof. Let

$$
\begin{aligned}
f(z) & =\int_{T} \frac{1}{(1-\bar{x} z)^{2}} d \mu(x) \\
& =\int_{T}\left(\sum_{n=0}^{\infty}(n+1) \bar{x}^{n+1} z^{n}\right) d \mu(x) \\
& =\sum_{n=0}^{\infty}(n+1) \hat{\mu}(n+1) z^{n} .
\end{aligned}
$$

So

$$
F(z)=\sum_{n=1}^{\infty} \hat{\mu}(n) z^{n}=C[\mu](z)-C[\mu](0) .
$$

The proof is reversible to obtain the converse.

Proposition C. Let $F=\int^{z} f$. Then the following are equivalent:

(i)

$$
\sup _{0 \leq r<1}\|[F(r x) \cdot d m(x)]\|_{M(T) / \bar{H}_{0}^{1}}<\infty .
$$

(ii) There is a measure $\mu \in M(T)$ such that $f=l[\mu]$.

(iii) There is a measure $\nu \in M(T)$ such that $F=C[\nu]$. Moreover, $[\nu]$ is a weak-* limit point of

$$
\{[F(r x) d m(x)]: 0<r<1\} \subseteq \frac{M(T)}{\bar{H}_{0}^{1}} .
$$

Proof. (ii) and (iii) are equivalent by the preceding lemma with

$$
\nu=\mu-\hat{\mu}(0) \cdot d m \text {. }
$$

The implication (i) $\Rightarrow$ (iii) is the Banach-Alagolu theorem for $M(T) /\left(H_{0}^{1}\right)^{-}$ $=\left(H^{\infty}(T) \cap C^{0}\right)^{*}$. Given (i), there exists a measure $\nu$ and a sequence $r_{n} \rightarrow 1$ such that

$$
\left[F\left(r_{n} x\right) d m(x)\right] \rightarrow[\nu],
$$

weak-*. In particular,

$$
\begin{aligned}
\int_{T} \frac{d \nu(x)}{(1-\bar{x} z)^{2}} & =\lim _{n \rightarrow \infty} \int_{T} \frac{F\left(r_{n} x\right) d m(x)}{(1-\bar{x} z)^{2}} \\
& =\lim _{n \rightarrow \infty} f\left(r_{n} z\right)=f(z) .
\end{aligned}
$$


Finally, assume (iii). Then

$$
f(z)=\sum_{k=0}^{\infty}(k+1) \hat{\nu}(k+1) z^{k} .
$$

Therefore,

$$
F(r x)^{\wedge}(n)=r^{n} \hat{\nu}(n), \quad \forall n \geq 0 .
$$

Let $P$ be an analytic polynomial

$$
P(x)=\sum_{k=0}^{N} a_{k} x^{k} .
$$

By the standard duality pairing,

$$
\begin{aligned}
\langle[\nu]-[F(r x)], P\rangle & \equiv \int_{T} P(\bar{x})(d \nu(x)-F(r x) d m(x)) \\
& =\sum_{k=0}^{N}\left(1-r^{k}\right) \hat{\nu}(k) a_{k}
\end{aligned}
$$

approaches zero as $r \rightarrow 1$. Therefore, $[F(r x) d m(x)] \rightarrow[\nu]$, weak- $*$, as $r \rightarrow 1$, and the boundedness of $\{\|[F(r x) d m(x)]\|: r<1\}$ follows from the Banach-Alagolu theorem.

We are now in a position to make the connection between the integral representations and local $H^{p}$ properties.

Let $\chi_{E}$ denote the characteristic function of a set $E$.

Proposition D. Let $g=C[\nu], \varepsilon>0$ be given and $l$ be a closed arc in $T$. If $g \in H_{\mathrm{loc}}^{1}(\Delta ; k l)$, where $k=1+\varepsilon$, then $\chi_{l} \nu$ is absolutely continuous.

The following argument uses two lemmas whose proofs we postpone to the next section.

Proof. According to Proposition C, (a subnet of)

$$
\{[F(r x) d m(x)]: r<1\} \text { converges weak-* to }[\nu] \text {. }
$$

We will ignore the indication of a subnet, since a slightly more careful proof of the proposition would actually show that $[\nu]$ is the only limit point.

Lemma A. $g \in H_{\text {loc }}^{1}(\Delta ; k l)$ implies that

$$
\left\{\chi_{k l}(x) g(r x): r<1\right\} \text { converges to } \chi_{k l}(x) g(x) \text { in } L^{1}(T) \text {. }
$$

Convergence in $L^{1}(T)$ is stronger than norm convergence in $M(T) /\left(H_{0}^{1}\right)^{--}$ since

$$
\frac{M(T)}{\bar{H}_{0}^{1}}=\frac{L^{1}(T)}{\bar{H}_{0}^{1}} \oplus M_{s}(T),
$$

where $M_{s}(T)$ denotes the space of measures singular with respect to Lebesgue measure. 
Then, combining (7) and (8) gives the result:

(9)

$\left\{\left[\chi_{T / l}(x) g(r x) d m(x)\right]: r<1\right\}$ converges weak- * to $\left[\nu(x)-\chi_{l}(x) g(x) d m(x)\right]$.

[Note: $\chi_{T / l}(x)=1-\chi_{l}(x)$, for all $x \in T$.]

Clearly,

$$
\begin{aligned}
h_{r}(z) & \equiv C\left[\chi_{T \backslash k l}(x) g(r x) d m(x)\right](z) \\
& =\int_{T \backslash k l} \frac{g(r x) d m(x)}{1-\bar{x} z}
\end{aligned}
$$

extends across $l$ (by the same formula) to a function analytic off $l$. Moreover, by Proposition $\mathrm{C}$ again,

$$
\sup _{0 \leq r<1}\left\|\left[\chi_{T \backslash k l}(x) g(r x) \cdot d m(x)\right]\right\| \equiv M<\infty .
$$

Therefore, if $z \notin T \backslash k l$, for every $r<1$,

$$
\left|h_{r}(z)\right| \leq \frac{1}{\operatorname{dist}(z ; T \backslash k l)} M .
$$

Now since $\left|h_{r}(z)\right| \quad(r<1)$ is locally uniformly bounded off $T \backslash k l$, there is a function $h$, analytic off $T \backslash k l$, and a sequence $r_{n}$, increasing to 1 , such that $h_{r_{n}}(z) \rightarrow h(z)$ uniformly on compacta. Since point evaluations are continuous linear functionals, it follows from (9) that

$$
\left.h\right|_{\Delta}=C\left[\nu(x)-\chi_{k l}(x) \cdot g(x) d m(x)\right] .
$$

In other words,

$$
C\left[\nu(x)-\chi_{k l}(x) \cdot g(x) d m(x)\right]
$$

extends analytically across $k l$.

Lemma B. If $C[\lambda]$ extends analytically across an arc $A$, then $\chi_{A} \cdot \lambda$ is absolutely continuous.

According to the lemma,

$$
\chi_{k l} \cdot\left(\nu-\chi_{l} \cdot g \cdot d m\right)=\chi_{k l} \cdot \nu-\chi_{l} \cdot g \cdot d m
$$

is absolutely continuous, and therefore so is $\chi_{k l} \nu$.

We are now able to prove our main theorems.

Theorem A. Let $l[\mu]$ be univalent.

(i) There is at most one atomic point for $\mu$.

(ii) The continuous component of $\mu$ is absolutely continuous.

Proof. Let

$$
g=\int_{0}^{z} l[\mu]=C[\mu-\hat{\mu}(0) d m] .
$$

Then, according to Proposition A

$$
g \in H_{\mathrm{loc}}^{1}(\Delta ; \zeta),
$$


for all but at most one point $\zeta_{0} \in T$. Now, let $l$ be any closed arc in $T$ not containing $\zeta_{0}$. Proposition D implies that

$$
\chi_{l} \cdot(\mu-\hat{\mu}(0) d m)
$$

is absolutely continuous. Thus, the singular part of $\mu\left(\mu_{s}\right)$ is concentrated in $(T \backslash l)^{-}$. Letting

$$
l_{n}=\left\{\zeta \in T:\left|\zeta-\zeta_{0}\right| \geq \frac{1}{n}\right\}
$$

and $n \rightarrow \infty$, shows that $\mu_{s}$ is concentrated at $\zeta_{0}$.

Theorem B. Let $C[\nu]$ be univalent.

(i) There are at most two atomic points for $\nu$.

(ii) The continuous component of $\nu$ is absolutely continuous.

Proof. According to Proposition A,

$$
C[\nu] \in H_{\mathrm{loc}}^{1}(\Delta ; \zeta)
$$

for all but at most two exceptional points $\zeta_{1}, \zeta_{2}$. By exhausting each of the components of $T \backslash\left\{\zeta_{1}, \zeta_{2}\right\}$ as in the above proof, we get the result that $\nu_{s}$ is concentrated in $\left\{\zeta_{1}, \zeta_{2}\right\}$.

\section{TWo LEMMAS}

In this section, we include the proofs of the two lemmas left from the proof of Proposition D. Each is a straightforward adaptation of well-known results.

Lemma B. Let $l$ be a closed arc in T. Suppose that

$$
f \in H_{\mathrm{loc}}^{p}(\Delta ;(1+\varepsilon) l), \quad p<\infty .
$$

Then

$$
\chi_{l}(x) \cdot f(r x) \stackrel{r \rightarrow 1}{\longrightarrow} \chi_{l}(x) \cdot f(x)
$$

in $L^{p}(T)$.

Proof. Let $\Omega_{l}$ be as in the proof of Proposition B, and $\phi: \Delta \rightarrow \Omega_{1}$ be a Riemann map with $\phi(0)=0$. By the Gauthier-Hengartner result, $f \in H^{p}\left(\Omega_{l}\right)$ or, equivalently, $f(\phi) \in H^{p}(\Delta)$. Therefore,

$$
f(\phi(r x)) \stackrel{r \rightarrow 1}{\longrightarrow} f(\phi(x))
$$

in $L^{p}(T)$.

Let $\left(t_{n}\right)$ be any sequence increasing to 1 . Since $f$ is a convex mapping, there exists a sequence $\left(r_{n}\right)$ increasing to 1 such that $t_{n} \phi(\Delta) \subseteq \phi\left(r_{n} \Delta\right)$.

Therefore, by Littlewood's subordination principle (see, e.g., [1]),

$$
\begin{aligned}
\int_{T}\left|f\left(t_{n} \phi(x)\right)\right|^{p} d m(x) & \leq \int_{T}\left|f\left(\phi\left(r_{n} x\right)\right)\right|^{p} d m(x) \\
& \leq \int_{T}|f(\phi(x))|^{p} d m(x)<\infty .
\end{aligned}
$$


Now, by the change of variable $\alpha=\phi(x)$ and dominated convergence, we get

$$
\lim _{t \rightarrow 1} \int_{\partial \Omega_{l}}|f(t \alpha)|^{p} \frac{1}{\left|\phi \cdot\left(\phi^{-1}(\alpha)\right)\right|}|d \alpha|=\int_{\partial \Omega_{l}}|f(\alpha)|^{p} \frac{1}{\left|\phi \cdot\left(\phi^{-1}(\alpha)\right)\right|}|d \alpha| .
$$

Since $f(t \alpha)$ converges pointwise a.e. to $f(\alpha)$,

$$
|f(t \alpha)| \cdot \frac{1}{\left|\phi \cdot\left(\phi^{-1}(\alpha)\right)\right|^{1 / p}} \stackrel{t \rightarrow 1}{\longrightarrow}|f(\alpha)| \cdot \frac{1}{\left|\phi \cdot\left(\phi^{-1}(\alpha)\right)\right|^{1 / p}}
$$

in $L^{p}(T)$.

The result then follows by noting that

$$
\left|\phi \cdot\left(\phi^{-1}(\alpha)\right)\right|^{1 / p}
$$

is $C^{\infty}(T)$, and therefore

$$
\chi_{l}(\alpha)\left|\phi \cdot\left(\phi^{-1}(\alpha)\right)\right|^{1 / p}
$$

is a multiplier of $L^{p}$.

Corollary (Lemma A). Let $l$ be a closed arc in $T$. If $C[\mu]$ extends continuously to $l$, then $\chi_{l} \mu$ is absolutely continuous.

Proof. Since $C[\mu]$ is bounded at each point of $l$, it is locally $H^{1}$ at each point of $l$. Therefore, $\chi_{l} \mu \in L^{1}(T)$ by the previous lemma.

\section{REFERENCES}

1. P. L. Duren, Theory of $H^{p}$ spaces, Academic Press, New York, 1970.

2. __ Univalent functions, Springer-Verlag, New York, 1983.

3. P. M. Gauthier and W. Hengartner, Local harmonic majorants of functions subharmonic in the unit disk, J. Analyse Math. 26 (1973), 405-417.

4. W. K. Hayman, Multivalent functions, Cambridge Univ. Press, Cambridge, 1958.

5. T. H. MacGregor, Analytic and univalent functions with integral representations involving a complex measure, Indiana Univ. Math. J. 36 (1987), 109-130.

6. W. Rudin, Functional analysis, McGraw-Hill, New York, 1973.

Department of Mathematics, University of Tennessee at Chattanooga, Chattanooga, TENNESSEE 37403 